\title{
Differing psychologically-derived clusters in people with chronic low back pain are associated with different multidimensional profiles.
}

Authors:

Martin Rabey

M.Manip.Th.

School of Physiotherapy and Exercise Science, Curtin University, Western Australia

martin.rabey@curtin.edu.au

Associate Professor Anne Smith

$\mathrm{PhD}$

School of Physiotherapy and Exercise Science, Curtin University, Western Australia

Anne.Smith@curtin.edu.au

Dr. Darren Beales

$\mathrm{PhD}$

School of Physiotherapy and Exercise Science, Curtin University, Western Australia

D.Beales@curtin.edu.au

Associate Professor Helen Slater

$\mathrm{PhD}$ 
School of Physiotherapy and Exercise Science, Curtin University, Western Australia

H.Slater@curtin.edu.au

Professor Peter O’Sullivan

$\mathrm{PhD}$

School of Physiotherapy and Exercise Science, Curtin University, Western Australia

P.Osullivan@curtin.edu.au

\section{Corresponding Author:}

Martin Rabey

School of Physiotherapy and Exercise Science

Curtin University of Technology

GPO Box U1987

Perth, Western Australia 6845

Tel: 0061421176663

E-mail: martin.rabey@gmail.com

\section{Conflicts of interest and sources of funding}

The authors declare that no conflicts of interest exist.

Martin Rabey was supported during his Doctor of Philosophy degree, of which this paper forms a part, by a Manipulation Association of Chartered Physiotherapists Doctoral Award, Chartered Society of Physiotherapy Charitable Trust, Curtin University Postgraduate Scholarship and Australian Postgraduate Award.

Darren Beales was supported by a National Health and Medical Research Council of Australia Early Career Research Fellowship. 


\begin{abstract}
Objectives

To explore the existence of subgroups in a cohort with chronic low back pain $(n=294)$ based upon data from multiple psychological questionnaires, and profile subgroups on data from multiple dimensions.
\end{abstract}

\title{
Methods
}

Psychological questionnaires considered as indicator variables entered into latent class analysis included: Depression, Anxiety, Stress scales, Thought Suppression and Behavioural Endurance subscales (Avoidance Endurance questionnaire), Chronic Pain Acceptance questionnaire (short-form), Pain Catastrophising Scale, Pain Self-Efficacy questionnaire, Fear-Avoidance Beliefs questionnaire. Multidimensional profiling of derived clusters included: demographics, pain characteristics, pain responses to movement, behaviours associated with pain, body perception, pain sensitivity and health and lifestyle factors.

\section{Results}

Three clusters were derived. Cluster $1(23.5 \%)$ was characterised by low cognitive and affective questionnaire scores, with the exception of fear-avoidance beliefs. Cluster $2(58.8 \%)$ was characterised by relatively elevated thought suppression, catastrophizing and fear-avoidance beliefs, but lower pain self-efficacy, depression, anxiety and stress. Cluster $3(17.7 \%)$ had the highest scores across cognitive and affective questionnaires.

Cluster 1 reported significantly lower pain intensity and bothersomeness than other clusters.

Disability, stressful life events and low back region perceptual distortion increased progressively from Cluster 1 to Cluster 3 while mindfulness progressively decreased. Clusters 2 and 3 had more people with an increase in pain following repeated forward and backward spinal bending, and more people with increasing pain following bending, than Cluster 1. Cluster 3 had significantly greater lumbar 
pressure pain sensitivity, more undiagnosed comorbid symptoms and more widespread pain than other clusters.

\section{Discussion}

Clinical implications relating to presentations of each cluster are postulated.

\section{Key words}

Chronic low back pain, psychological, multidimensional, subgrouping

\section{INTRODUCTION}

There is growing evidence that a broad range of psychological factors are associated with pain and disability, and may mediate the relationship between pain and disability, in people with chronic low back pain (CLBP) ${ }^{1-2}$. Unhelpful psychological factors include both cognitive (e.g. kinesiophobia, pain catastrophizing, endurance behaviours, low acceptance, low pain self-efficacy) and affective factors (e.g. depressed mood, anxiety, stress) ${ }^{3-4}$. There is evidence that rather than acting independently, psychological factors overlap in people with CLBP, leading to calls to consider them as broader constructs (e.g. pain-related distress) ${ }^{3,5}$.

Understanding the influences of psychological factors has led to psychologically-based interventions for people with CLBP. However, to date treatment outcomes for these interventions in people with CLBP are moderate at best ${ }^{6}$, possibly reflecting the heterogeneity of study samples or because other dimensions associated with CLBP (e.g. pain characteristics, health, lifestyle, tissue sensitivity, movement) are not targeted by these interventions ${ }^{7}$. To facilitate better understanding of the complexities of CLBP, a research priority is to determine subgroups of people with CLBP with different clinical profiles ${ }^{8}$, to facilitate development of tailored interventions and improve outcomes ${ }^{9}$. To achieve this, analysis should consider a range of factors from multiple, relevant dimensions ${ }^{7}$, and to minimise bias CLBP subgroups should be "data-driven": identified within large, diverse samples using unsupervised statistical techniques derived from cross-sectional data, independent of previously determined associations or potential outcomes ${ }^{10}$. 
While CLBP subgroups have been statistically-derived previously using data from psychological measures ${ }^{11-12}$, these studies have both examined a limited number of different measures making it unclear which psychological factors are most important for deriving subgroups.

Where interventions have been tailored towards CLBP subgroups, derived predominantly from psychological factors, outcomes have still been suboptimal ${ }^{13-14}$ suggesting other dimensions may contribute to persistence of the disorder, and be important for optimising targeted management. For example, unhelpful cognitive and affective factors have been associated with greater pain intensity levels during repeated lifting ${ }^{15}$, more widespread pain ${ }^{16}$, increased local and widespread pain sensitivity ${ }^{17-18}$, impaired motor control ${ }^{19}$, protective ${ }^{20}$, avoidance and endurance behaviours ${ }^{21}$, and distorted perception of the low back region ${ }^{22}$. These associations highlight the complexity of multidimensional interactions underlying the lived experience of $\mathrm{CLBP}^{23}$, the limited nature of multidimensional profiling of psychologically-derived subgroups to date, and the potential importance of profiling subgroups across multiple interacting dimensions.

Therefore the aims of this study were:

1) Using latent class analysis of a broad range of psychological indicator variables, to determine the existence and number of clusters in a cohort of people with axial CLBP.

2) To profile identified clusters according to demographics, pain characteristics, health and lifestyle factors, body perception, tissue sensitivity, pain responses to movement and behaviours associated with pain.

\section{MATERIALS AND METHODS.}

This research was conducted in accordance with the Declaration of Helsinki and approved by the Human Research Ethics Committees of Curtin University, Royal Perth Hospital and Sir Charles Gairdner Hospital in Perth, Western Australia. 


\section{Study population}

This cross-sectional study involved people with axial CLBP $(n=294 ; 57.1 \%$ female; median age 50 years old) recruited from the aforementioned public metropolitan hospitals (1.4\%); private metropolitan pain management and general practice clinics (1.0\%) and physiotherapists (20.1\%), and via multi-media advertisements in metropolitan and rural Western Australia (77.6\%).

Potential participants contacted one researcher (MR) by telephone or e-mail. They were subsequently sent a self-report inclusion / exclusion criteria screening questionnaire. Ambiguous responses to any criteria were clarified by telephone.

Inclusion criteria were: aged 18-70 years old; > 3 months duration of LBP; pain intensity of $\geq$ twopoints on a numeric rating scale (NRS) (0, "no pain"-10, "worst pain imaginable") in the past week; five-points scored on the Roland Morris Disability Questionnaire (RMDQ) ${ }^{24}$; a score of at least $60 \%$ LBP on the question ${ }^{25}$, "Which situation describes your pain over the past 4 weeks the best? $100 \%$ of the pain in the low back; $80 \%$ of the pain in the low back and $20 \%$ in the leg(s); $60 \%$ of the pain in the low back and $40 \%$ in the leg(s); $50 \%$ of the pain in the low back and $50 \%$ in the leg(s); $40 \%$ of the pain in the low back and $60 \%$ in the leg(s); or $20 \%$ of the pain in the low back and $80 \%$ in the leg(s)." This final question reliably differentiates dominant leg pain from dominant $\mathrm{LBP}^{25}$, minimizing the likelihood of recruitment of participants with radiculopathy.

Exclusion criteria were: previous extensive spinal surgery (greater than single level fusion / instrumentation or discectomy); spinal surgery within the past six months, serious spinal pathology (cancer, inflammatory arthropathy, acute yertebral fracture); diagnosed neurological disease; bilateral dorsal wrist / hand pain; pregnancy; inadequate command of English.

For included participants paper copies of all questionnaires were mailed for completion at their convenience at home (duration approximately 30 minutes). An appointment (duration 60-90 minutes) was made for them to attend the Pain Research Laboratory at Curtin University within approximately two weeks, for completion of the physical examination. Questionnaires were checked for missing data 
when the participant attended the study centre. All participants completed the following physical examination in this order: two-point discrimination, pain sensitivity testing (temporal summation; pressure, heat and cold pain thresholds) and repeated bending tasks.

Ethical approval was contingent upon not influencing participant's medication use, and therefore participants were allowed to continue all medications as prescribed.

\section{Indicator variables for derivation of psychologically-based subgroups}

Following extensive review of the literature, a broad range of individual psychological factors most commonly associated with pain and disability in people with CLBP were considered as indicator variables for entry into latent class analysis (LCA). Despite conceptual overlap between such variables ${ }^{3,5}$ subscales of each individual questionnaire considered have been shown to measure unique constructs ${ }^{26-30}$ and it is unknown which constructs may be most important for psychological subgroup derivation. While these variables may be associated with each other in a cohort as a whole, LCA allows derivation of subgroups based upon differing response patterns across the indicator variables. For example, while depression may be associated with fear-avoidance beliefs ${ }^{3}$, LCA may allow the derivation of a subgroup who exhibit low levels of depression but high levels of fearavoidance, which may be important for the future development of tailored interventions. The following variables were considered as indicator variables in LCA.

\section{Depression, anxiety and stress}

The short-form version of the Depression Anxiety Stress scales (DASS-21) ${ }^{31}$ is a valid and reliable questionnaire with three subscales, each containing seven statements evaluating depression, anxiety and stress symptoms. Each statement is rated on a 0 - 3 scale, and the score is doubled to give a score of 0 - 42 points per subscale, with higher scores reflecting greater symptoms. 


\section{Fear-avoidance beliefs}

The Fear Avoidance Beliefs questionnaire (FABQ) measures of fear of pain / re-injury. It is reliable ${ }^{30}$ and valid ${ }^{32}$. The physical activity (FABQ-PA subscale) contains four statements regarding fear of pain / re-injury for which participants indicate their level of agreement on a 0 - 6 scale giving a score of 0 - 24 points. The work subscale (FABQ-W) contains seven such statements giving a score of 0 42 points. Higher scores reflect higher fear avoidance beliefs.

\section{Endurance behaviours}

The Avoidance Endurance questionnaire (AEQ) ${ }^{21}$ is a valid and reliable measure of endurance behaviours. The Thought Suppression sub-scale (TSS) comprises four statements, which examine suppression of thoughts regarding pain. The Behavioural Endurance Sub-scale (BES) comprises 12 statements, which examine persistence behaviours. Participants are asked to respond to each statement such as, "I distract myself with physical activity," on a 0 - 6 scale from, "Never," to, "Always." A mean score is derived for each subscale, with higher scores reflecting greater thought suppression or behavioural endurance.

\section{Pain catastrophising}

The Pain Catastrophising scale (PCS) is a valid and reliable ${ }^{33}$ questionnaire examining a person's thoughts and feelings in terms of magnification, rumination, and helplessness about pain. On a 0 - 4 scale participants indicate the frequency at which they experience these different types of catastrophic thoughts described in 13 statements, giving a total score of 0 - 52 points, with higher scores reflecting greater pain catastrophising. The rumination subscale comprises four statements, the magnification subscale three statements, and the helplessness subscale six statements, giving scores of $0-16,0-12$ and 0 - 24 respectively.

\section{Pain self-efficacy}


The Pain Self-Efficacy Questionnaire (PSEQ) ${ }^{34}$ is a valid and reliable measure of a person's beliefs regarding their ability to undertake activities despite pain ${ }^{35-36}$. Participants rate how confident they are of undertaking actions described in 10 statements, on a 0 - 6 scale, giving a score of $0-60$ points, with higher scores indicating higher pain self-efficacy.

\section{Acceptance}

The short-form Chronic Pain Acceptance Questionnaire (CPAQ-8) ${ }^{37}$ is a valid and reliable measure of a person's ability to have ongoing pain without attempting to avoid or control it. Participants indicate their level of agreement with eight statements relating to acceptance of chronic pain, scored on a $0-6$ scale. It has two subscales (four statements each): pain willingness (not engaging in behaviours to avoid pain, particularly when this may limit functioning or reduce quality of life) and activity engagement (engaging in activities whilst in pain). There is therefore a score of $0-24$ points for each subscale, and an overall total of 48 points indicating greater acceptance.

\section{Multidimensional profiling variables}

CLBP is a multidimensional disorder ${ }^{23}$, therefore variables from multiple dimensions were considered as profiling variables, to be compared between subgroups. As per the indicator variables, profiling variables were selected based on established associations with CLBP, taken from the demographic / pain characteristic dimension, health and lifestyle dimensions, tissue sensitivity dimension and movement dimension.

\section{Demographics / pain characteristic dimension}

Assessment of the demographic / pain characteristic dimension considered age, sex, pain intensity, pain duration, CLBP-related disability, bothersomeness, and perceived risk of persistent pain.

Age and sex were collected for each participant.

Average pain intensity (during the past week) was determined using a valid and reliable NRS ( 0 (no pain) - 10 (worst pain imaginable) $)^{38}$. 
Duration of symptoms was determined by asking participants, "How long have you had your back pain for?" Responses were converted into months.

CLBP-related disability was measured using the Roland Morris Disability questionnaire (RMDQ) ${ }^{24}$, which examines the influences of LBP on physical activities during daily life. It is valid and reliable ${ }^{24,}$ 39. The RMDQ comprises 24 items, which the participant may tick to indicate whether the item is relevant to their presentation. Scores range from 0 - 24, with higher scores indicating higher disability.

The following single question was used as a measure of the bothersomeness of any reported CLBP, "Overall, how bothersome has your back pain been in the last 2 weeks?" Responses on a five-point scale from, "not at all," to, "extremely," were dichotomised with participants answering from, "not at all," to, "moderately," forming one group, and those answering, "very much," or, "extremely" forming another ${ }^{40}$.

To assess the participant's perceived risk of persistent pain a 0 - 10 scale from the valid and reliable Örebro Musculoskeletal Pain Questionnaire was used; anchored at one end by, "No risk," and at the other by, "Very large risk," for the question, "In your view, how large is the risk that your current pain may become persistent?" 41

\section{Health and lifestyle dimensions}

Assessment of the health and lifestyle dimensions considered stressful life events, sleep quality, mindfulness, physical activity levels, comorbidities, multiple pain sites, and perception of the low back region.

The self-perceived impact of stressful life events was measured on an NRS (0, "No stress," - 6, "Extreme stress") for the question, "In the past year, how would you rate the amount of stress in your life (at home and at work)?" This is a valid and reliable single question assessing life events and hassles ${ }^{42}$.

The Pittsburgh Sleep Quality index (PSQI) contains 17 questions examining sleep quality, quantity, disturbance and its effect on daily living. A scoring schema is described by the original authors, which 
generates a final score from $0-21$ points. If this score is above five points it suggests significant sleep disturbance. It is reliable and valid ${ }^{43}$.

The Mindful Attention Awareness Scale (MAAS) ${ }^{44}$ is a valid and reliable measure of mindfulness. It consists of 15 statements regarding mindfulness such as, "I break or spill things because of carelessness, not paying attention, or thinking of something else." Subjects are asked to rate the frequency with which these statements relate to their day-to-day activities on a 0 - 6 scale. A mean score is calculated, with higher scores indicating greater mindfulness.

For the short-form version of the International Physical Activity questionnaire (IPAQ) ${ }^{45}$ participants estimate the amount of activity they have done in the past seven days and the scoring guidelines ${ }^{45}$ allow calculation of the number of moderate and vigorous minutes of physical activity per week. It is valid and reliable ${ }^{46}$.

To assess the presence of comorbidities associated with CLBP, participants were asked to self-report whether they had a number of diagnosed medical conditions (specifically heart disease, diabetes, ulcer or stomach disease, anaemia or other blood disease, cancer, osteoarthritis, rheumatoid arthritis, fibromyalgia, hypertension, depression, neurological disorders, eczema, osteoporosis, incontinence or bladder problems, respiratory disorders, migraine or recurrent headache, irritable bowel syndrome, chronic fatigue syndrome, pelvic pain or vulvodynia, temporomandibular joint pain, hay fever or some other allergy, eating disorders, anxiety disorders, visual or hearing disorders, thyroid disorders) ${ }^{47-48}$ or undiagnosed symptoms (constipation, diarrhoea, palpitations, dizziness, chest pain, stomach discomfort, breathing difficulties, tiredness, flushes / heat sensations) ${ }^{49-50}$. Total counts of the number of diagnosed conditions ( 0 - 25) and undiagnosed symptoms $(0-9)$ were used for analysis.

Assessment of the all regions of the body where pain was perceived was undertaken by completion of a quantifiable body chart. A grid allowed a total count of squares (0 - 256) of the body chart containing any marking to be generated. This method is valid and reliable ${ }^{16}$. 
The Fremantle Back Awareness questionnaire (FreBAQ) ${ }^{51}$ examines patient perception of body schema in relation to the low back region. It consists of nine statements regarding perception of the lumbar region such as, "My back feels as though it is not part of the rest of my body," for which the participants indicate the degree of agreement with the statement using an NRS anchored at one end by, " 0 ," and, "Never," and at the other by, "4," and, "Always". There is a maximum score of 36 points, a higher score indicating higher perceptual dysfunction. This questionnaire demonstrates adequate reliability, construct and discriminative validity ${ }^{51}$.

\section{Tissue sensitivity dimension}

Psychophysical sensory tests were chosen to examine somatosensory submodalities mediated by different primary afferents (C, A delta, A beta) ${ }^{52}$, and assess central nervous system nociceptive and non-nociceptive processing ${ }^{53}$ and included assessment of two-point discrimination, temporal summation and pressure and thermal pain thresholds.

All participants were positioned prone during testing, which was undertaken in the same order with each subject beginning with the test deemed least likely to be provocative of pain, progressing to those more likely to be provocative. An experienced clinician (MR) undertook all testing.

All tests (pain thresholds, temporal summation, two-point discrimination) were undertaken in the area of maximal lumbar pain indicated by the participant ${ }^{54}$. Pain threshold testing (pressure, heat, cold) was also performed at the dorsal wrist joint line ${ }^{55}$ of a pain-free wrist (if both wrists were pain-free, the non-dominant wrist), with the participant's arm supported on the plinth. The wrist was tested before the lumbar region. Testing utilised standardised instructions aligned to the Standardised Evaluation of Pain ${ }^{56}$ or German Research Network on Neuropathic Pain QST protocol, as relevant ${ }^{57}$. Current best practice for quantitative sensory testing was adhered to ${ }^{58}$. Standard protocols for pain threshold testing include a 30-second inter-stimulus interval to reduce the likelihood of temporal summation ${ }^{59-60}$. Therefore, between temporal summation testing, and the application of each stimulus during pain threshold testing (pressure, heat and cold) 30-second inter-stimulus intervals were 
adopted. Testing of pressure pain thresholds prior to thermal pain thresholds, was also adopted to reduce the likelihood of increasing sensitisation with repeated testing ${ }^{61}$.

Two-point discrimination (TPD) was undertaken in the region of maximal lumbar pain only using the method described by Moberg ${ }^{62}$, updated by Luomajoki and Moseley ${ }^{63}$. Participants were instructed that a plastic calliper ruler (Aestheisometer, DanMic Global, San Jose, USA), would be used to gently touch their lower back region. The amount of pressure applied was just enough to cause the, "very first small blanching," around the calliper points ${ }^{62}$ (p.128). Each time they were touched they were instructed to tell the examiner whether they believed they felt one or two points of the calliper touching them by saying, "One," or, "Two." Participants were also able to state that they were unsure as to whether they had felt one or two points. If they were unsure, testing simply continued, the distance between the callipers having been altered. All applications of the calliper were with the points aligned horizontally ${ }^{63}$. The TPD threshold was taken as the minimum distance between the two calliper points at which the subject stated with certainty that they had been touched by two points rather than one. Both ascending and descending runs, where the distance between the calliper points were increased or decreased by $5 \mathrm{~mm}$ at a time respectively, were tested. A mean of three runs was used to calculate the threshold. "Trick" stimuli, where the callipers were applied at a distance that was out of sequence, or where only one point made contact, were randomly applied to minimise the chances of the participant guessing. The distance between the two points of the calliper ranged from 0 -10 centimetres.

Detection of perceived temporal summation was tested with a 26g Semmes-Weinstein nylon monofilament ${ }^{56}$. The participant was questioned whether the first application of the filament was painful. If so, they rated the pain intensity on the previously described NRS. If no pain was provoked, pain intensity for this stimulus was recorded as zero. The filament was then repeatedly applied $(1 \mathrm{~Hz}$, $30 \mathrm{sec}$ ). Participants rated the pain intensity again at the end of stimulation. Enhanced temporal summation was deemed to have occurred if participants perceived the initial stimulus as non-noxious, but it became noxious, increasing $\geq 2$ points on the NRS (equivalent to the minimum clinically 
important difference (MCID) ${ }^{64}$ ) during repeated stimulation; or if participants perceived the first stimulus as noxious, and pain intensity increased $\geq 2$ points during stimulation. A binary outcome of whether enhanced temporal summation did, or did not, occur was recorded.

Pressure pain threshold (PPT) was defined as the point when the sensation of pressure perceived by the participant changed to a sensation of pressure and pain ${ }^{57}$. PPT was tested using an algometer (probe size $1 \mathrm{~cm}^{2}$; Somedic $\mathrm{AB}$, Sweden). Pressure increased from $0 \mathrm{kPa}$, at $50 \mathrm{kPa} / \mathrm{s}$, until the subject indicated their PPT by pressing a button. The mean of three thresholds was used for analysis.

Heat pain threshold (HPT), defined as the temperature at which a sensation of warmth becomes the sensation of heat and pain ${ }^{57}$, was tested using the Thermotest (Somedic AB, Sweden; thermode contact area $2.5 \mathrm{~cm} \times 5 \mathrm{~cm}$ ). Testing began at $32{ }^{\circ} \mathrm{C}$, with the temperature increasing $1^{\circ} \mathrm{C} / \mathrm{s}$ until the participant detected their threshold and pressed a button, or the device's upper limit $\left(50^{\circ} \mathrm{C}\right)$ was reached. The mean of three thresholds was used for analysis.

Cold pain threshold (CPT) was defined as the point when the sensation of cold became the sensation of cold and pain ${ }^{57}$. Testing CPT utilised the aforementioned contact thermode. Testing began at 32 ${ }^{\circ} \mathrm{C}$, with the temperature decreasing $1{ }^{\circ} \mathrm{C} / \mathrm{s}$ until the participant detected their threshold and pressed a button, or the device's lower limit $\left(4^{\circ} \mathrm{C}\right)$ was reached. The mean of three thresholds was used for analysis.

\section{Movement dimension}

Assessment of the movement dimension incorporated two repeated spinal bending tasks, from which the following constructs were measured: pain provocation following repeated spinal bending, behaviours associated with pain and time taken to complete the tasks.

Participants were asked to perform the following two repeated spinal bending tasks: 
1. To complete 20 forward spinal bends, with the cue to pick up a pencil from the floor.

Repeated forward bending ( 20 repetitions) is a valid and reliable test of pain provocation for people with CLBP ${ }^{65}$.

Participants received standardised instructions to pick up a pencil that was placed on the floor in front of them. This counted as one forward bend. They then placed the pencil back on the floor, which counted as the second forward bend. They repeated this until a total of 20 forward bends was reached. Participants were told that they could undertake this task however they wished, and at whatever speed they wished.

2. To complete 20 backward spinal bends, with the cue to view a marker placed on the ceiling behind the participant.

Repeated backward bending was included as it forms a common component of the physical examination for CLBP, and to determine whether pain provocation may be influenced in a directional manner ${ }^{66}$.

Participants received standardised instructions to take sight of the marker placed on the ceiling approximately $60 \mathrm{~cm}$ behind them however they wished, at whatever speed they wished, but without turning around, and then to return to neutral before repeating the task up to a total of 20 times.

Standardised instructions were reiterated if the participant subsequently questioned whether they should perform the task in a certain manner. Participants were instructed that there would be a brief pause every five repetitions, during both tasks, to ask them to rate their pain intensity (see pain intensity during repeated movements below).

Participants were able to refuse to undertake these movements, or decline to complete the full 20 repetitions should they feel that their pain became too great, or fear exacerbation of symptoms because of these movements. The number of repetitions completed was recorded. 
Video recordings were made of the repeated movements using two iPads (4th Generation) (Apple, California, USA) (1080p HD video recording) mounted on tripods. One iPad was directly in front of the participant; the second was repositioned to optimise the lateral view of the participant's lumbar region. These views allowed adequate visualisation to enable the coding of behaviours associated with pain provocation ${ }^{20,67}$ (See below).

From these tasks it was determined whether the participant exhibited pain provocation following repeated spinal bending, and behaviours associated with pain using the methods detailed below:

Assessment of whether repeated movements influenced perceived pain intensity was undertaken by asking participants to rate their pain intensity on a valid and reliable NRS ( 0 (no pain) - 10 (worst pain imaginable) $)^{38}$, using a protocol adapted from ${ }^{15}$, allowing determination of whether pain increased with movement repetition. Participants rated their pain intensity before commencing the movements, then following every five repetitions. A change score was determined by subtracting the pain intensity score after the last set of repetitions completed (maximum 20) from the baseline score (adapted from ${ }^{15}$ ). Pain was subsequently deemed to have increased only if it had increased by the MCID (twopoints ${ }^{64}$. Participants were subgrouped as follows: no increase in pain $(<2$-point change, both directions); increase in pain forward bending only ( $\geq 2$-point change following forward bending, $<2$ point change following backward bending); increase in pain backward bending only ( $\geq 2$-point change following backward bending, <2-point change following forward bending); bidirectional increase in pain ( $\geq 2$-point change both directions). Subgroup membership was used for profiling.

Assessment of behaviours associated with pain was undertaken by viewing video playback, using both angles, and repeated viewings where necessary, to obtain a total count of behaviours witnessed during the first five bends of each movement task ${ }^{20,67}$. No minimum duration was stipulated for any behaviour ${ }^{20}$. Assessment of these behaviours demonstrates good intra-rater agreement ${ }^{67}$.

Protective behaviours included:

a) guarding - abnormally slow or rigid movements 
b) bracing - using a limb for extra support during movement

c) rubbing or holding the affected area

Communicative behaviours included:

a) grimacing, or other facial expressions of pain

b) sighing, grunting, moaning etc.

From the video playback, the time (seconds), taken to complete the first five bends in each direction, was also recorded. This commenced at the initiation of the first bend, and was completed at the participant's return to a neutral standing position after the fifth bend.

\section{STATISTICAL ANALYSIS}

\section{Data management prior to latent class analysis}

The number of participants with missing data for each variable are detailed in Tables 1-5. For questionnaires missing data management was undertaken as suggested in original manuscripts, where described. Otherwise, if one item was missing the imputed average of other items was used in the calculation of the questionnaire total, with the exception of the Pittsburgh Sleep Quality index for which omission of certain single items means it is impossible to generate the total score.

Questionnaire totals were coded as missing, when two or more items were missing. Only $76.2 \%$ of the sample was currently working, therefore data from the FABQ (Work) were excluded from analysis. Before LCA, the PSEQ score was reversed, so that a higher score reflected worse psychological functioning across all indicator variables. Two participants declined to undertake the movement task in both directions. For subgrouping purposes, these directions of movement were coded as provocative for these participants. For behaviours associated with pain, and time taken to complete bending tasks, these participants were coded as missing. Testing for temporal summation revealed 28 (9.5\%) participants who perceived the initial stimulus as non-noxious, but it increased $\geq$ two-points on an NRS during stimulation. Four participants (1.4\%) perceived the initial stimulus as noxious, and 
deemed the pain intensity to increase by $\geq$ two-points during stimulation. These two groups were combined for future analysis $(\mathrm{n}=32,10.9 \%)$.

\section{Latent class analysis}

LCA was used to estimate the number of clusters based upon responses to the psychological indicator variable questionnaires. LCA is a probabilistic form of cluster analysis using maximum likelihood estimation, which has advantages over traditional distance-based cluster procedures by allowing statistical evaluation of the optimal number of clusters, inclusion of variables with differing measurement types, and calculation of classification probabilities for each participant ${ }^{68-69}$. Sample size requirements for LCA are not definitive, but depend upon many factors including the size and number of true latent classes, and the model complexity (number, type and correlation of indicator variables). However, simulation studies of LCA suggest >200 participants are preferable when using continuous variables ${ }^{70}$ and $>300$ participants with dichotomous variables ${ }^{71}$

LCA was performed using 12 psychological indicator variables. A sample size of 300 participants allows accurate latent class derivation based upon inclusion of 12 indicator variables ${ }^{71}$. Models containing between one and five clusters were estimated. One thousand random starts were estimated to reduce the possibility of local solutions. Models were developed with examination of unique loglikelihood solutions, degree of contributions of each indicator variable, and residual correlations within classes. Examination of model fit involved comparison of model fit statistics (Akaike information criterion (AIC), Bayesian information criterion (BIC)) and posterior probability diagnostics. Cluster membership for each participant was the determined based upon posterior probability. To ensure the skewed nature of the ordinal data for some scales did not influence the latent class estimations, models were also estimated using quantiles of each indicator variable. As this procedure generated similar solutions the solution derived from raw data is presented, with increased confidence in validity of parameter estimates. 


\section{Multidimensional profiling}

Between-cluster differences in indicator and profiling variables were examined using analysis of variance for normally-distributed variables, Kruskal-Wallis one-way analysis of variance for variables with skewed data, and chi-squared analysis for dichotomous data.

No correction for multiple comparisons was undertaken. As LCA is an exploratory technique for deriving clusters within a sample ${ }^{69}$, we maintained $p$-values such that while there was a greater chance of type I error, there was less chance of type II error ${ }^{72}$.

Latent class analysis was undertaken using Latent GOLD 4.5 (Statistical Innovations Inc., Belmont, USA), and all other statistical procedures performed using Stata 13.1 (Statacorp, Texas, USA).

\section{RESULTS}

\section{Latent class analysis}

Initial latent class models included 12 psychological indicator variables (Table 1), but due to an inability to obtain a unique log-likelihood solution, only those eight indicators contributing substantially to the models $\left(\mathrm{R}^{2}>0.3\right)$ were retained. The indicator variables retained in the model were: DASS depression subscale, DASS anxiety subscale, DASS stress subscale, TSS of the AEQ, PCS rumination, PCS magnification, PCS helplessness and PSEQ (reverse scored). Using these eight indicators, models containing between one and five clusters were estimated.

The three and four cluster models were examined in detail. The three cluster model had the most unique log likelihood, and was supported by the BIC statistic (One cluster model: 14008, two cluster model: 13619, three cluster model: 13515, four cluster model: 13550, five cluster model: 13579). There was also an increase in classification error associated with the four cluster model (0.07) compared to the three cluster model (0.05). However, the four cluster model was supported by examining the results of the conditional bootstrapping procedure, which suggested that the four cluster model was a better fit than the three cluster model $(p=<.001)$, and by the AIC statistic (one cluster model: 13927, two cluster model: 13454, three cluster model: 13265, four cluster model: 
13215, five cluster model: 13159). Therefore, for both the three and four cluster models, cluster profiles were calculated using raw data from each retained indicator variable. These profiles, combined with their graphical representation, and the relative distributions of cluster membership, were visually inspected and compared between models. Interpretation of the cluster profiles in both models was informed by comparison to published data from healthy controls and, where available, from CLBP samples. In the four cluster model, there were two clusters with generally low scores across all indicator variables. These two clusters equated to splitting the lowest scoring cluster from the three cluster model. It was determined that retaining these 2 clusters would not facilitate the clinical interpretation of the cluster profiles. Therefore, the final solution chosen was the three cluster model, this being the most parsimonious.

For the three cluster model the mean (SD) probability of membership was $.95(.09), .94(.10)$ and .93 (.11) for Clusters 1, 2 and 3 respectively. This exceeds the recommended minimum for model adequacy of $.7^{73}$. The odds of correct classification were 63.6, 11.3 and 60.5 for Clusters 1,2 and 3 respectively. Larger measures indicate better assignment accuracy, and a minimum value of 5 has been suggested to represent high assignment accuracy ${ }^{73}$. The classification error of the three cluster model was acceptable at 0.05 .

Figure 1 displays the three cluster solution. Cluster $1(23.5 \%)$ was characterised by low scores across all retained indicator variables. Cluster $2(58.8 \%)$ had relatively low scores related to negative affect (particularly the DASS depression and anxiety scores), with moderately high scores on the other indicator variables. Cluster $3(17.7 \%)$ was characterised by high scores across all retained indicator variables.

Table 1 shows descriptive statistics for questionnaire scores for each cluster, for each of the eight retained indicator variables and four variables that did not contribute significantly to the final model. There were significant differences between clusters for each of the variables except the two CPAQ-8 subscales. 


\section{Multidimensional profiling}

Descriptive data are detailed in Tables 2-5 for each profiling variable including demographics, pain characteristics, health and lifestyle factors, tissue sensitivity, pain responses to movement and behaviours associated with pain.

\section{Demographic / pain characteristic dimension}

There was a significant between-group difference for median age, with Cluster 1 being significantly older than Cluster 3. Cluster 1 reported significantly lower pain intensity in the past week, and had a significantly lower proportion of people who deemed their CLBP very or extremely bothersome than the other clusters. There was a significant progressive increase in disability levels from Cluster 1 to Cluster 3. (Table 2)

\section{Health and lifestyle dimensions}

There was a significant progressive increase in reported stressful life events from Cluster 1 to Cluster 3. Conversely, there was a significant progressive decrease in mindfulness from Cluster 1 to Cluster 3 . There was a significant progressive increase in FreBAQ scores (indicating greater distortion of perception of the low back region) from Cluster 1 to Cluster 3. Cluster 3 also had a significantly greater number of undiagnosed comorbid symptoms and more widespread pain (filled-in body chart squares) than Clusters 1 and 2. (Table 3)

\section{Tissue sensitivity dimension}

Cluster 3 had significantly greater pressure pain sensitivity at the lumbar spine than Clusters 1 and 2 (Table 4).

\section{Movement dimension}

Comparing pain responses to movement Cluster 1 was significantly different from Clusters 2 and 3, having a greater proportion of people with no increase in pain following repeated movements, and a lesser proportion of people with bidirectional increases in pain following repeated movement. 
Behaviours associated with pain (protective / guarding), and time taken to complete the bending tasks, were examined separately for forward and backward bending. However, there were no significant differences found, therefore Table 5 contains summed data for these variables. (Table 5)

\section{DISCUSSION}

We derived three psychological clusters from a broad range of psychological measures in this CLBP cohort. Cluster $1(23.5 \%)$ was characterised by low scores across all retained indicator variables. Compared to Cluster 1, cognitive scores for Cluster 2 (58.8\%) included elevated thought suppression and catastrophizing, and lower pain self-efficacy, while affective scores (depression, anxiety, stress) remained relatively low. Cluster $3(17.7 \%)$ demonstrated higher scores across all retained indicator variables.

Data from our clusters can be compared to questionnaire cut-off scores and normative data. Depression, anxiety and stress can be classified as normal ${ }^{31}$ for Clusters 1 and 2 , and are similar to healthy controls, except stress which appears slightly elevated in Cluster $2^{74}$. Cluster 3 may be classified as having extremely severe depression, severe anxiety and moderate stress ${ }^{31}$, with elevated scores compared to healthy controls ${ }^{74}$.

Cluster 1 had lower median thought suppression (0.2) than the mean (SD) $(3.5(1.0))$ reported in a previous LBP cohort ${ }^{75}$, reflecting their low-scoring psychological profile. Pain catastrophising scores were similar to healthy controls in Cluster 1, while in Cluster 2 scores appeared elevated but due to large standard deviations may still be within normal limits ${ }^{74}$. In Cluster 3, pain catastrophising was elevated compared to healthy controls ${ }^{74}$, being the only cluster with a median score $>30$ indicating clinically-relevant catastrophising ${ }^{76}$. For pain self-efficacy, Cluster 3 scored similarly to a CLBP cohort attending pain management ${ }^{34}$, Cluster 2 scored similarly to people seeking treatment in primary care ${ }^{77}$, while the median score for Cluster 1 was significantly higher (e.g. greater selfefficacy) ${ }^{2}$. Fear-avoidance beliefs were elevated in all clusters compared to healthy controls ${ }^{78}$, however, Cluster 3 scored notably higher than other CLBP cohorts ${ }^{79}$. 
Although fear avoidance beliefs and behavioural endurance did not contribute discriminatory information to cluster analysis, and therefore were not retained indicator variables, they differed between clusters. Contrastingly, pain acceptance was not retained and did not differ across clusters. Our findings are presented by considering psychologically-derived clusters, their multidimensional profiling and clinical implications.

\section{Psychologically-derived clusters}

This study included the broadest range of psychological measures to date for the derivation of clusters in people with CLBP, where previous studies tend to cluster on a limited number of factors such as coping strategies, affect, somatisation or attitudes towards pain ${ }^{11-12}$. This study included factors used in previous but less comprehensive clustering studies (e.g. depression, anxiety), but added novel factors (e.g. thought suppression).

In addition our study used LCA allowing optimised assignment of individuals to clusters and statistical evaluation of the optimal number of clusters ${ }^{69}$. LCA is more accurate at identifying clusters than the k-means cluster analysis ${ }^{68}$ used in isolation in two previous cluster analysis studies involving people with CLBP ${ }^{11-12}$.

While direct comparison with other psychological cluster analysis studies is complicated by variability in the measures and clustering techniques used, similarities exist suggesting possible common psychological presentations. Numerous studies deriving two, three or four cluster solutions describe broadly similar low and high-scoring psychologically-derived clusters (acute / sub-acute ${ }^{80-82}$, variable duration (49\% CLBP) ${ }^{83}, \mathrm{CLBP}^{11-12}$ ). Consistent with our findings, lower-scoring CLBP clusters presented with lower anxiety and depression; higher pain self-efficacy and positive coping strategies ${ }^{11-12}$. Higher-scoring clusters had higher anxiety and depression, and more negative coping strategies ${ }^{11-12}$. Further comparison with these studies is limited by use of differing questionnaires (e.g. Coping Strategies questionnaire, Survey of Pain Attitudes ${ }^{12}$ ).

Three studies, with three or four cluster solutions, derived intermediate clusters scoring relatively low for affect (predominantly depression), and relatively high on fear-avoidance beliefs ${ }^{80-82}$; also having 
intermediate levels of pain intensity and disability broadly consistent with Cluster 2 . These studies involved acute / sub-acute cohorts, suggesting characteristics shown by Cluster 2 may develop at an early stage. The identification of Cluster 2 where depression and anxiety were normal, but cognitive variables such as thought suppression, fear-avoidance beliefs and pain catastrophizing were elevated and pain self-efficacy was lower, appears clinically important as these factors have previously been associated with increased pain and disability in CLBP, potentially warranting tailored management ${ }^{5}$ 84-86

\section{Multidimensional profiling}

The broad range of profiling variables in this study is novel, having been limited in other cluster analysis studies to demographics, pain characteristics, employment, healthcare utilisation and comorbidities ${ }^{11-12,80-83}$. Such novel multidimensional profiling adds a new level of validation to the derived clusters ${ }^{10}$. Consistent with our findings, previous studies have reported higher pain intensity and disability, and more widespread pain and comorbidities associated with higher versus lowerscoring psychological clusters ${ }^{11-12}$. Although statistically significant, differences in pain intensity between clusters are below the MCID, so may not be clinically important ${ }^{64}$, while the difference in disability between Clusters 1 and 3 is clinically important, being greater than the MCID on the RMDQ $^{87}$.

Cluster 1 had the most localised pain, lowest pain intensity (5.1 / 10 on an NRS), least bothersomeness (27.5\% rated their CLBP very / extremely bothersome) and lowest disability levels (RMDQ score: 6). Cluster 1 had the highest proportion of participants with no increase in pain following repeated bending, and lowest proportion with increased pain following repeated forward and backward bending (bidirectional). While comparing pain sensitivity in these clusters with healthy controls should be undertaken cautiously due to different test sites / protocols and large standard deviations, Cluster 1 appears within normal limits for pain sensitivity ${ }^{57,88-90}$. Cluster 1 had the lowest levels of stressful life events and undiagnosed comorbid symptoms, and highest mindfulness. While 
they had low scores for distorted body perception compared to other clusters and a previously reported CLBP cohort, they still scored higher than healthy controls ${ }^{51}$.

Cluster 2 had more widespread pain, and higher pain intensity (6.0) and bothersomeness (57.8\%) than Cluster 1, intermediate levels of disability (RMDQ score: 9) and the most even spread of pain provocation responses following repeated bending. Pain sensitivity appeared within normal limits ${ }^{57}$, ${ }^{88-90}$. However, Cluster 2 had intermediate levels of stressful life events, mindfulness and distorted body perception.

Cluster 3 (17.7\%) had higher pain intensity (6.2) and bothersomeness (67.3\%) than Cluster 1, the most widespread pain and greatest disability (RMDQ score: 12). They had the highest proportion of participants with increased pain following repeated forward bending, and forward and backward bending (bidirectional), and lowest proportion with no pain increase following repeated bending. Cluster 3 had a significantly lower lumbar PPT suggesting increased sensitivity compared to normative data ${ }^{88-89}$, the highest levels of undiagnosed comorbid symptoms, and stressful life events and lowest mindfulness. Compared to Clusters 1 and 2, greater undiagnosed comorbid symptoms and stressful life events, combined with their higher psychological profile, suggests increased allostatic load may be relevant to Cluster 3's presentation ${ }^{48}$. Cluster 3 had the greatest distortion of body perception, higher than a previous CLBP cohort ${ }^{51}$. Consistent with this study body perceptual distortion has been positively associated with pain intensity and pain catastrophizing ${ }^{51}$ possibly through altered interoception ${ }^{91}$. This cluster is similar to a previously reported CLBP subgroup demonstrating increased pain sensitivity, higher DASS scores, greater sleep disturbance and exaggerated / prolonged pain responses to movement ${ }^{17}$.

There were no significant differences between clusters for protective behaviours despite previous associations between these behaviours and pain intensity and disability ${ }^{20}$, which did differ between clusters. Two-point discrimination, reflecting body schema within $\mathrm{S}^{92}$, did not differ between clusters despite differing body perception ${ }^{51}$, suggesting these measures reflect differing perceptual constructs. Sleep quality was similar across all clusters despite poor sleep being previously associated 
with increased stress ${ }^{93}$ and depression ${ }^{94}$ which did differ between clusters. However, PSQI scores represented significant sleep disturbance ${ }^{43}$ across all clusters, consistent with other people with CLBP 95.

\section{Clinical implications}

As this study was cross-sectional, the nature and direction of associations between and within clusters is unknown. It is also unknown whether cluster membership predicts outcomes, however previous research involving psychologically-derived clusters would suggest this is likely ${ }^{80-82}$.

Although our clusters were psychologically-derived, the differing multidimensional profiles of each cluster are consistent with a contemporary multidimensional view of CLBP, and may provide greater direction for targeted care ${ }^{7}$. While the literature documents multiple psychological subgrouping studies, few have targeted treatments to psychologically-derived subgroups. Where matched treatments have been offered, long-term outcomes have been similar to control or unmatched treatments ${ }^{13-14,96}$. One limitation of these approaches may be the lack of targeting other dimensions such as pain responses to movement, distorted body perception and increased pain sensitivity. While there is early evidence suggesting management tailored towards findings from structured examination of multidimensional profiles in people with CLBP may offer improved outcomes compared to usual care $^{9}$, further research is needed.

While speculative, targeted management for Cluster 1 could involve challenging fear-avoidance beliefs and protective behaviours, while improving sensorimotor perception and sleep quality. In Cluster 2, management could target cognitive factors such as pain catastrophising and pain-self efficacy, and sensorimotor disturbances as well as enhancing stress resilience and sleep quality. Cluster 3's multidimensional profile suggests tailored multidisciplinary management might target psychological factors, sleep quality and sensorimotor disturbances in parallel with appropriate pharmacological management ${ }^{97}$ and addressing comorbidities ${ }^{98}$. 


\section{Strengths and limitations}

Most participants were recruited via advertisements, facilitating generalizability to the wider community. Only participants with dominant $\mathrm{CLBP}^{25}$ were included, minimizing the likelihood of participants having radiculopathy. Other inclusion criteria included reporting pain intensity $\geq$ twopoints on an NRS and scoring $\geq$ five-points on the RMDQ, which may have influenced cluster membership, reducing the size of the low-scoring cluster.

Clinical measures chosen to facilitate translation into practice and reduce participant burden, were not necessarily gold standard measurements (e.g. PSQI scores versus polysomnography). Gold standard measurements may facilitate further understanding of multidimensional profiles, and subsequent management directions.

The exploratory nature of this study meant that $p$-values were not corrected for multiple comparisons. Readers should interpret $p$-values in this light, and future research should further examine associations between the clusters and their multidimensional profiles.

\section{References}

1. Hayden JA, Dunn KM, van der Windt DA, et al. What is the prognosis of back pain? Best Practice \& Research Clinical Rheumatology. 2010; 24: 167-179.

2. Lee H, Hubscher M, Moseley G, et al. How does pain lead to disability? A systematic review and meta-analysis of mediation studies in people with back and neck pain. Pain. 2015; 156: 988-997.

3. Campbell P, Bishop A, Dunn KM, et al. Conceptual overlap of psychological constructs in low back pain. Pain. 2013; 154: 1783-1791.

4. Pincus T and McCracken LM. Psychological factors and treatment opportunities in low back pain. Best Practice \& Research Clinical Rheumatology. 2013; 27: 625-635.

5. Foster NE, Thomas E, Bishop A, et al. Distinctiveness of psychological obstacles to recovery in low back pain patients in primary care. Pain. 2010; 148: 398-406. 
6. Ramond-Roquin A, Bouton C, Gobin-Tempereau A-S, et al. Interventions focusing on psychosocial risk factors for patients with non-chronic low back pain in primary care-a systematic review. Family Practice. 2014; 31: 379-388.

7. Rusu A, Boersma K, Turk D. Reviewing the concept of subgroups in subscute and chronic pain and the potential of customising treatments. In: Hasenbring M, Rusu A, Boersma $\mathrm{K}$ et al., eds From Acute to Chronic Back Pain: Risk Factors, Mechanisms and Clinical Implications. Oxford: Oxford University Press, 2012:485-511.

8. Costa L, Koes BW, Pransky G, et al. Primary care research priorities in low back pain. An update. Spine. 2013; 38: 148-156.

9. Vibe Fersum K, O'Sullivan P, Skouen JS, et al. Efficacy of classification-based cognitive functional therapy in patients with non-specific chronic low back pain: A randomized controlled trial. European Journal of Pain. 2013; 17: 916-928.

10. Kent P, Keating J and Leboeuf-Yde C. Research methods for subgrouping low back pain. BMC Medical Research Methodology. 2010; 10: 62.

11. Viniol A, Jegan N, Hirsch O, et al. Chronic low back pain patient groups in primary care - a cross sectional cluster analysis. BMC Musculoskeletal Disorders. 2013; 14: 294.

12. Strong J, Large R, Ashton R, et al. A new zealand replication of the ipam clustering model for low back patients. Clinical Journal of Pain. 1995; 11: 296-306.

13. Bergbom S, Flink IL, Boersma K, et al. Early psychologically informed interventions for workers at risk for pain-related disability: Does matching treatment to profile improve outcome? Journal of Occupational Rehabilitation. 2014; 24: 446-457.

14

15. Sullivan MJL, Thibault P, Andrikonyte J, et al. Psychological influences on repetition-induced summation of activity-related pain in patients with chronic low back pain. Pain. 2009; 141: 70-78. 16. Öhlund C, Eek C, Palmblad S, et al. Quantified pain drawing in subacute low back pain: Validation in a nonselected outpatient industrial sample. Spine. 1996; 21: 1021-1030. 
17. O'Sullivan P, Waller R, Wright A, et al. Sensory characteristics of chronic non-specific low back pain: A subgroup investigation. Manual Therapy. 2014; 19: 311-318.

18. Campbell CM and Edwards RR. Mind-body interactions in pain: The neurophysiology of anxious and catastrophic pain-related thoughts. Translational Research. 2009; 153: 97-101.

19. Lewis $\mathrm{S}$, Holmes $\mathrm{P}$, Woby $\mathrm{S}$, et al. The relationships between measures of stature recovery, muscle activity and psychological factors in patients with chronic low back pain. Manual Therapy. 2012; 17: 27-33.

20. Sullivan MJL, Thibault P, Savard A, et al. The influence of communication goals and physical demands on different dimensions of pain behavior. Pain. 2006; 125: 270-277.

21. Hasenbring MI, Hallner D, Klasen B, et al. Pain-related avoidance versus endurance in primary care patients with subacute back pain: Psychological characteristics and outcome at a 6-month followup. Pain. 2012; 153: 211-217.

22. Beales D, Lutz A, Thompson J, et al. Disturbed body perception, reduced sleep, and kinesiophobia in subjects with pregnancy-related persistent lumbopelvic pain and moderate levels of disability: An exploratory study. Manual Therapy.

23. Simons LE, Elman I and Borsook D. Psychological processing in chronic pain: A neural systems approach. Neuroscience \& Biobehavioral Reviews. 2014; 39: 61-78.

24. Roland M and Morris R. A study of the natural history of back pain. Part i: Development of a reliable and sensitive measure of disability in low-back pain. Spine. 1983; 8: 141-144.

25. Wai E, Howse K, Pollock W, et al. The reliability of determining “leg dominant pain'. The Spine Journal. 2009; 9: 447-453

26. Parkitny L, McAuley J, Walton D, et al. Rasch analysis supports the use of the depression, anxiety and stress scales to measure mood in groups but not in individuals with chronic low back pain. Journal of Clinical Epidemiology. 2012; 65: 189-198.

27. Fish RA, Hogan MJ, Morrison TG, et al. Willing and able: A closer look at pain willingness and activity engagement on the chronic pain acceptance questionnaire (cpaq-8). The Journal of Pain. 2013; 14: 233-245. 
28. Van Damme S, Crombez G, Bijttebier P, et al. A confirmatory factor analysis of the pain catastrophizing scale: Invariant factor structure across clinical and non-clinical populations. Pain. 2002; 96: 319-324.

29. Hasenbring MI, Hallner D and Rusu AC. Fear-avoidance- and endurance-related responses to pain: Development and validation of the avoidance-endurance questionnaire (AEQ). European Journal of Pain. 2009; 13: 620-628.

30. Waddell G, Newton M, Henderson I, et al. A fear-avoidance beliefs questionnaire (FABQ) and the role of fear-avoidance beliefs in chronic low back pain and disability. Pain. 1993; 52: 157-168.

31 Lovibond S and Lovibond P. Manual for the depression anxiety stress scales. Sydney: School of Psychology, University of New South Wales, 1995.

32. George S, Valencia C and Beneciuk J. A psychometric investigation of fear-avoidance model measures in patients with chronic low back pain. Journal of Orthopaedic and Sports Physical Therapy. 2010; 40: 197-205.

33. Sullivan M, Bishop S and Pivik J. The pain catastrophizing scale: Development and validation. Psychological Assessment. 1995; 7: 524-532.

34. Nicholas M. The pain self-efficacy questionnaire: Taking pain into account. European Journal of Pain. 2007; 11: 153-163.

35. Kaivanto KK, Estlander AM, Moneta GB, et al. Isokinetic performance in low back pain patients: The predictive power of the self-efficacy scale. Journal of Occupational Rehabilitation. 1995; 5: 8799.

36. Asghari A and Nicholas MK. Pain self-efficacy beliefs and pain behaviour. A prospective study. Pain. 2001; 94: 85-100.

37. Fish RA, McGuire B, Hogan M, et al. Validation of the chronic pain acceptance questionnaire (CPAQ) in an internet sample and development and preliminary validation of the CPAQ-8. Pain. 2010; 149: 435-443.

38. Dworkin RH, Turk DC, Farrar JT, et al. Core outcome measures for chronic pain clinical trials: Immpact recommendations. Pain. 2005; 113: 9-19. 
39. Kuijer W, Brouwer S, Dijkstra P, et al. Responsiveness of the Roland/Morris disability questionnaire: Consequences of using different external criteria. Clinical Rehabilitation. 2005; 19: 488-495.

40. Dunn K and Croft P. Classification of low back pain in primary care: Using "bothersomeness" to identify the most severe cases. Spine. 2005; 30: 1887-1892.

41. Linton S and Boersma K. Early identification of patients at risk of developing a persistent back problem: The predictive validity of the örebro musculoskeletal pain questionnaire. Clinical Journal of Pain. 2003; 19: 80-86.

42. Littman A, White E, Satia J, et al. Reliability and validity of 2 single-item measures of psychosocial stress. Epidemiology. 2006; 17: 398-403.

43. Buysse DJ, Reynolds CF, Monk TH, et al. The pittsburgh sleep quality index: A new instrument for psychiatric practice and research. Psychiatry Research. 1989; 28: 193-213.

44. Brown K and Ryan R. The benefits of being present: Mindfulness and its role in psychological well-being. Journal of Personality and Social Psychology 2003; 84: 822-848.

45

46. Craig C, Marshall A, Sjostrom M, et al. International physical activity questionnaire: 12-country reliability and validity. Medicine and Science in Sports and Exercise. 2003; 35: 1381-1395.

47. Beales DJ, Smith AJ, O'Sullivan PB, et al. Low back pain and comorbidity clusters at 17 years of age: A cross-sectional examination of health-related quality of life and specific low back pain impacts. Journal of Adolescent Health. 2012; 50: 509-516.

48. Dominick CH, Blyth FM and Nicholas MK. Unpacking the burden: Understanding the relationships between chronic pain and comorbidity in the general population. Pain. 2012; 153: 293304.

49. Tschudi-Madsen H, Kjeldsberg M, Natvig B, et al. A strong association between nonmusculoskeletal symptoms and musculoskeletal pain symptoms: Results from a population study. BMC Musculoskeletal Disorders. 2011; 12: 285. 
50. Hagen E, Svensen E, Eriksen H, et al. Comorbid subjective health complaints in low back pain. Spine. 2006; 31: 1491-1495.

51. Wand BM, James M, Abbaszadeh S, et al. Assessing self-perception in patients with chronic low back pain: Development of a back-specific body-perception questionnaire. Journal of Back and Musculoskeletal Rehabilitation. 2014; 27: 463-473.

52. Jensen TS and Baron R. Translation of symptoms and signs into mechanisms in neuropathic pain. Pain. 2003; 102: 1-8.

53. Cruz-Almeida Y and Fillingim R. Can quantitative sensory testing move us closer to mechanismbased pain management? Pain Medicine. 2014; 15: 61-72.

54. Ho S, Tong H, Attaman J, et al. Central sensitization of pressure pain threshold in low back pain subjects. . American Journal of Physical Medicine and Rehabilitation. 2006; 85: 260.

55. Blumenstiel K, Gerhardt A, Rolke R, et al. Quantitative sensory testing profiles in chronic back pain are distinct from those in fibromyalgia. Clinical Journal of Pain. 2011; 27: 682-690.

56. Scholz J, Mannion RJ, Hord DE, et al. A novel tool for the assessment of pain: Validation in low back pain. PLoS Medicine. 2009; 6: e1000047.

57. Rolke R, Baron R, Maier C, et al. Quantitative sensory testing in the german research network on neuropathic pain (DFNS): Standardized protocol and reference values. Pain. 2006; 123: 231-243. 58. Backonja MM, Attal N, Baron R, et al. Value of quantitative sensory testing in neurological and pain disorders: Neupsig consensus. Pain. 2013; 154: 1807-1819.

59. Brennum J, Kjeldsen M, Jensen K, et al. Measurements of human pressure-pain thresholds on fingers and toes. Pain. 1989; 38: 211-217.

60. Graven-Nielsen T, Wodehouse T, Langford RM, et al. Normalization of widespread hyperesthesia and facilitated spatial summation of deep-tissue pain in knee osteoarthritis patients after knee replacement. Arthritis \& Rheumatism. 2012; 64: 2907-2916.

61. Gröne E, Crispin A, Fleckenstein J, et al. Test order of quantitative sensory testing facilitates mechanical hyperalgesia in healthy volunteers. The Journal of Pain. 2012; 13: 73-80. 
62. Moberg E. Two point discrimination test. A valuable part of hand surgical rehabilitation.

Scandinavian Journal of Rehabilitation Medicine. 1990; 22: 127-134.

63. Luomajoki H and Moseley GL. Tactile acuity and lumbopelvic motor control in patients with back pain and healthy controls. British Journal of Sports Medicine. 2011; 45: 437-440.

64. Salaffi F, Stancati A, Silvestri CA, et al. Minimal clinically important changes in chronic musculoskeletal pain intensity measured on a numerical rating scale. European Journal of Pain. 2004; 8: 283-291.

65. Brouwer S, Reneman MF, Dijkstra PU, et al. Test-retest reliability of the Isernhagen work systems functional capacity evaluation in patients with chronic low back pain. Journal of Occupational Rehabilitation. 2003; 13: 207-218.

66. O'Sullivan P. Diagnosis and classification of chronic low back pain disorders: Maladaptive movement and motor control impairments as underlying mechanism. Manual Therapy. 2005; 10: 242255.

67. Keefe FJ and Block AR. Development of an observation method for assessing pain behavior in chronic low back pain patients. Behavior Therapy. 1982; 13:363-375.

68. Magidson J and Vermunt J. Latent class models for clustering: A comparison with k-means. Canadian Journal of Marketing Research. 2002; 20: 37-44.

$69^{\wedge}$. Collins L and Lanza S. Latent class and latent transition analysis. With applications in the social, behavioral, and health sciences. Hoboken, New Jersey: John Wiley \& Sons, Inc, 2010. 70. Nylund K, Asparouhov A and Muthén B. Deciding on the number of classes in latent class analysis and growth mixture modeling: A monte carlo simulation study. Structural Equation Modeling. 2007; 14: 535-569.

71. Swanson S, Lindenberg K, Bauer S, et al. A monte carlo investigation of factors influencing latent class analysis: An application to eating disorder research. International Journal of Eating Disorders. 2012; 45: 677-684.

72. Armstrong R. When to use the bonferroni correction. Ophthalmic \& Physiological Optics. 2014; 34: 502-508. 
73. Nagin D. Group-based modeling development. Cambridge, MA, USA: Harvard University Press, 2005.

74. Mitchell T, O’Sullivan P, Smith A, et al. Biopsychosocial factors are associated with low back pain in female nursing students: A cross-sectional study. International Journal of Nursing Studies. 2009; 46: 678-688.

75. Scholich SL, Hallner D, Wittenberg RH, et al. The relationship between pain, disability, quality of life and cognitive-behavioural factors in chronic back pain. Disability and Rehabilitation. 2012; 34: 1993-2000.

76. SULLIVAN M. The Pain Catastrophising Scale. User Manual [Online]. 1995. Available at: http://sullivan-painresearch.mcgill.ca/pdf/pcs/PCSManual_English.pdf Accessed January 23rd 2015.

77. Costa L, Maher C, McAuley J, et al. Self-efficacy is more important than fear of movement in mediating the relationship between pain and disability in chronic low back pain. European Journal of Pain. 2011; 15: 213-219.

78. Dedering Å and Börjesson T. Assessing fear-avoidance beliefs in patients with cervical radiculopathy. Physiotherapy Research International. 2013; 18: 193-202.

79. Wertli MM, Rasmussen-Barr E, Weiser S, et al. The role of fear avoidance beliefs as a prognostic factor for outcome in patients with nonspecific low back pain: A systematic review. The Spine Journal. 2014; 14: 816-836.e814.

80. Boersma K and Linton S. Screening to identify patients at risk: Profiles of psychological risk factors for early intervention. Clinical Journal of Pain. 2005; 21: 38 - 43.

81. Barons MJ, Griffiths FE, Parsons N, et al. Matching patients to an intervention for back pain: Classifying patients using a latent class approach. Journal of Evaluation in Clinical Practice. 2014; 20: 544-550.

82. Hirsch O, Strauch K, Held H, et al. Low back pain patient subgroups in primary care - pain characteristics, psychosocial determinants and health care utilization. Clinical Journal of Pain. 2015; In Press: 
83. Beneciuk JM, Robinson ME and George SZ. Subgrouping for patients with low back pain: A multidimensional approach incorporating cluster analysis and the start back screening tool. The Journal of Pain. 2015; 16: 19-30.

84. Cook AJ, Brawer PA and Vowles KE. The fear-avoidance model of chronic pain: Validation and age analysis using structural equation modeling. Pain. 2006; 121: 195-206.

85. Thibault P, Loisel P, Durand M-J, et al. Psychological predictors of pain expression and activity intolerance in chronic pain patients. Pain. 2008; 139: 47-54.

86. Crombez G, Vlaeyen JWS, Heuts PHTG, et al. Pain-related fear is more disabling than pain itself: Evidence on the role of pain-related fear in chronic back pain disability. Pain. 1999; 80: 329-339. 87. Stratford PW, Binkley J, Solomon P, et al. Defining the minimum level of detectable change for the Roland-Morris questionnaire. Physical Therapy. 1996; 76: 359-365.

88. Neziri AY, Scaramozzino P, Andersen OK, et al. Reference values of mechanical and thermal pain tests in a pain-free population. European Journal of Pain. 2011; 15; 376-383.

89. Pfau DB, Krumova EK, Treede R-D, et al. Quantitative sensory testing in the german research network on neuropathic pain (DFNS): Reference data for the trunk and application in patients with chronic postherpetic neuralgia. Pain. 2014; 155: 1002-1015.

90. Magerl W, Krumova EK, Baron R, et al. Reference data for quantitative sensory testing (QST): Refined stratification for age and a novel method for statistical comparison of group data. Pain. 2010; 151: 598-605.

91. Tsay A, Allen TJ, Proske U, et al. Sensing the body in chronic pain: A review of psychophysical studies implicating altered body representation. Neuroscience \& Biobehavioral Reviews. 2015; 52:

221-232.

92. Pleger B, Tegenthoff M, Ragert $\mathrm{P}$, et al. Sensorimotor returning in complex regional pain syndrome parallels pain reduction. Annals of Neurology. 2005; 57: 425-429.

93. Åkerstedt T. Psychosocial stress and impaired sleep. Scandinavian Journal of Work, Environment and Health. 2006; 32: 493-501. 
94. Boakye P, Olechowski C, Rashiq S, et al. A critical review of neurobiological factors involved in the interactions between chronic pain, depression, and sleep disruption. Clinical Journal of Pain.

2015; In Press:

95. Marty M, Rozenberg S, Duplan B, et al. Quality of sleep in patients with chronic low back pain: A case-control study. European Spine Journal. 2008; 17: 839-844.

96. Hill JC, Whitehurst DGT, Lewis M, et al. Comparison of stratified primary care management for low back pain with current best practice (start back): A randomised controlled trial. The Lancet. 2011; 378: 1560-1571.

97. Baron R, Hans G and Dickenson AH. Peripheral input and its importance for central sensitization. Annals of Neurology. 2013; 74: 630-636.

98. Hartvigsen J, Natvig B and Ferreira M. Is it all about a pain in the back? Best Practice \& Research Clinical Rheumatology. 2013; 27: 613-623.

Figure 1: Final three cluster model derived using latent class analysis, with all psychological questionnaire scores standardised to a common scale (0-1).

DASS depression etc. - Depression Anxiety Stress scales depression score etc., TSS - Thought Suppression subscale score, PCS rumination etc. - Pain Catastrophising scale rumination score etc., PSEQ - Pain Self-Efficacy questionnaire score (reversed)

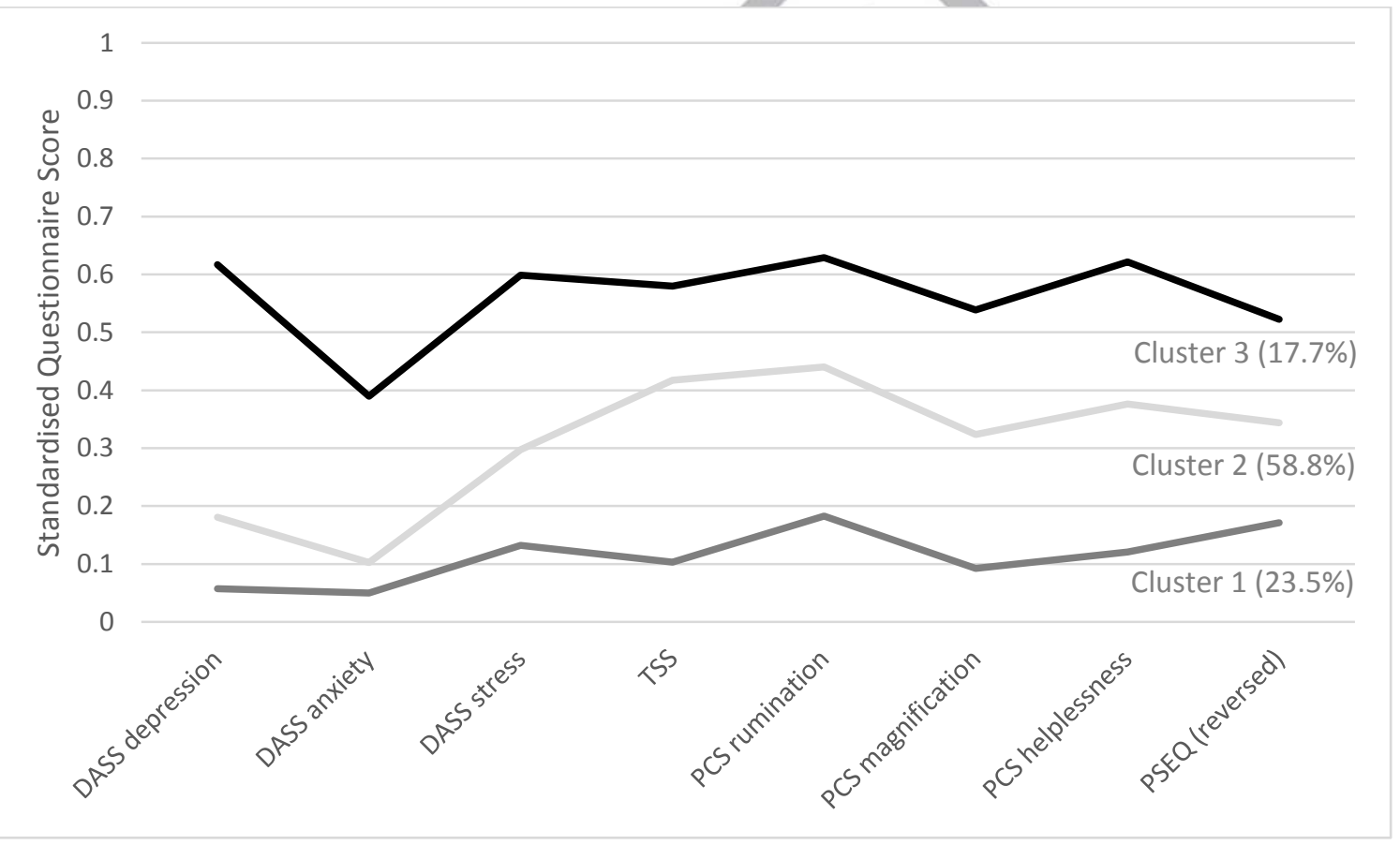


Table 1: Psychological indicator variable questionnaire scores for the three clusters derived using latent class analysis

\begin{tabular}{|c|c|c|c|c|}
\hline Indicator variable & $\begin{array}{c}\text { Cluster } 1 \\
(\mathrm{n}=69,23.5 \%)\end{array}$ & $\begin{array}{c}\text { Cluster } 2 \\
(\mathrm{n}=173,58.8 \%)\end{array}$ & $\begin{array}{c}\text { Cluster } 3 \\
(\mathrm{n}=52,17.7 \%)\end{array}$ & $p$-value \\
\hline $\begin{array}{l}\text { DASS depression score* } \\
\text { Median (IQR) } \\
(\min , \max )\end{array}$ & $\begin{array}{c}2^{a}(0,4) \\
(0,10)\end{array}$ & $\begin{array}{c}6^{\mathrm{b}}(2,12) \\
(0,24)\end{array}$ & $\begin{array}{c}28^{\mathrm{c}}(20,34) \\
(0,42)\end{array}$ & $<.001^{1}$ \\
\hline $\begin{array}{l}\text { DASS anxiety score* } \\
\text { median (IQR) } \\
\text { (min, } \max )\end{array}$ & $\begin{array}{l}2^{\mathrm{a}}(0,4) \\
(0,10)\end{array}$ & $\begin{array}{c}4^{b}(2,6) \\
(0,18)\end{array}$ & $\begin{array}{c}16^{\mathrm{c}}(8,24) \\
(0,42)\end{array}$ & \\
\hline $\begin{array}{l}\text { DASS stress score* } \\
\text { median (IQR) } \\
(\text { min, max })\end{array}$ & $\begin{array}{c}6^{a}(2,8) \\
(0,16)\end{array}$ & $\begin{array}{c}12^{\mathrm{b}}(8,18) \\
(0,36)\end{array}$ & $4^{\mathrm{c}}($ & $<.00$ \\
\hline $\begin{array}{l}\text { FABQ-PA } \\
\text { mean }(\mathrm{SD}) \\
(\min , \max )\end{array}$ & $\begin{array}{c}12.5^{\mathrm{a}}(6.1) \\
(0,24)\end{array}$ & 14. & $7.7^{\mathrm{c}}(4.7)$ & $<.001^{2}$ \\
\hline $\begin{array}{l}\text { TSS* } \\
\text { median (IQR) } \\
(\min , \max )\end{array}$ & $\begin{array}{l}.2^{\mathrm{a}}(0,1) \\
(0,2.5)\end{array}$ & $2.8^{\mathrm{b}}(1.5,3.5)^{3}$ & $\begin{array}{c}3.6^{\mathrm{c}}(2.8,4.4) \\
(0,6)\end{array}$ & $<.0011^{1}$ \\
\hline $\begin{array}{l}\text { BES } \\
\text { mean }(\mathrm{SD}) \\
(\min , \max )\end{array}$ & $6 \mathrm{a}(1)$ & $\begin{array}{l}3.2^{\mathrm{b}}(.9) \\
(0,5.9)\end{array}$ & $\begin{array}{c}3.3^{\mathrm{b}}(1.2) \\
(0,6)\end{array}$ & $<.001^{2}$ \\
\hline $\begin{array}{l}\text { PCS (Rumination)* } \\
\text { median (IQR) } \\
\text { (min, max) }\end{array}$ & $\begin{array}{l}2^{\mathrm{a}}(1,5) \\
(0,9)\end{array}$ & $\begin{array}{c}7^{\mathrm{b}}(4,10) \\
(0,16)\end{array}$ & $\begin{array}{c}10^{\mathrm{c}}(7,13)^{3} \\
(0,16)\end{array}$ & $<.001^{1}$ \\
\hline
\end{tabular}


PCS (Magnification)*

median (IQR)
$1^{\mathrm{a}}(0,2)$
$4^{\mathrm{b}}(2,5)$
$7^{\mathrm{c}}(4,9)^{3}$

(min, max)
$(0,4)$
$(0,12)$
$(0,12)$

$<.001^{1}$

PCS (Helplessness)*

median (IQR)

$3^{\mathrm{a}}(1,4)$

$9^{b}(6,12)$

$15^{\mathrm{c}}(12,19)^{3}$
$(0,7)$
$(0,21)$
$(2,24)$

(min, max)

PSEQ*

median (IQR)

(min, max)

CPAQ (Pain willingness)

mean (SD)

$(0,21)$

$(0,20)$

(min, max)

CPAQ (Activity

engagement)

$18(15,20)$

$17(12.5,22)$

median (IQR)

$(0,24)$

$(6,24)$

(min, max)

\footnotetext{
${ }^{1}$ Kruskal-Wallis one-way analysis of variance; ${ }^{2}$ analysis of variance; ${ }^{3}$ missing in one case

* Indicates indicator variable included in final three cluster model

${ }^{a, b, c}$ Superscripted letters define significantly different groups, i.e. results with different letters are significantly different

DASS - Depression Anxiety Stress scales; FABQ-PA - Fear-Avoidance Beliefs questionnaire - Physical activity; CPAQ - Chronic Pain Acceptance Questionnaire; TSS - Thought Suppression Subscale; BES Behavioural Endurance subscale; PCS - Pain Catastrophising scale; PSEQ - Pain Self-Efficacy Questionnaire
} 
Table 2: Demographic and pain characteristic data for the three clusters derived using latent class analysis

\begin{tabular}{lcccc}
\hline & Cluster 1 & Cluster 2 & Cluster 3 & $p$-value \\
Variable & $(\mathrm{n}=69,23.5 \%)$ & $(\mathrm{n}=173,58.8 \%)$ & $(\mathrm{n}=52,17.7 \%)$ & \\
\hline
\end{tabular}

Demographics
Age, years
$56^{\mathrm{a}}(41,63)$
$50^{\mathrm{ab}}(39,60)$
$45^{\mathrm{b}}(29,55)$
median (IQR) (min,max)
$(20,70)$
$(18,70)$
$(19,68)$
$.002^{1}$
Female
41 (59.4)
$93(53.8)$
34 (65.4)
$.30^{2}$
$\mathrm{n}(\%)$
Pain characteristics
Pain intensity (NRS)
mean $(\mathrm{SD})(\min , \max )$
$5.1^{\mathrm{a}}(2.0) \quad 6.0^{\mathrm{b}}(1.8)$
$<.001^{3}$
Duration of CLBP, months
$144(60,300)$
$120(36,240)$
$96(36,150)$
median (IQR) (min,max)
$(6,540)$
$(3,720)^{4}$
$(4,516)$
$.10^{1}$
RMDQ Score
median (IQR) (min,max)
$6^{\mathrm{a}}(6,9)$
$9^{\mathrm{b}}(7,13)$
$12^{\mathrm{c}}(9,16.5)$
$(5,18)$
$(5,21)$
$(5,24)$
$<.001^{1}$
Bothersomeness (very / extremely)
$19^{\mathrm{a}}(27.5)$
$100^{\mathrm{b}}(57.8)$
$35^{\mathrm{b}}(67.3)$
$<.001^{2}$
n $(\%)$ 
Perceived risk of persistent pain

(NRS)
$8(7,10)$
$9(8,10)$
$9(8,10)$
$(3,10)$
$(3,10)$
$(6,10)$

median (IQR) (min,max)

\footnotetext{
${ }^{1}$ Kruskal-Wallis one-way analysis of variance; ${ }^{2} \chi^{2}$ analysis; ${ }^{3}$ analysis of variance; ${ }^{4}$ missing in four cases

a,b,c Superscripted letters define significantly different groups, i.e. results with different letters are significantly different
}

NRS - numeric rating scale; RMDQ - Roland Morris Disability questionnaire

Table 3: Health and lifestyle data for the three clusters derived using latent class analysis

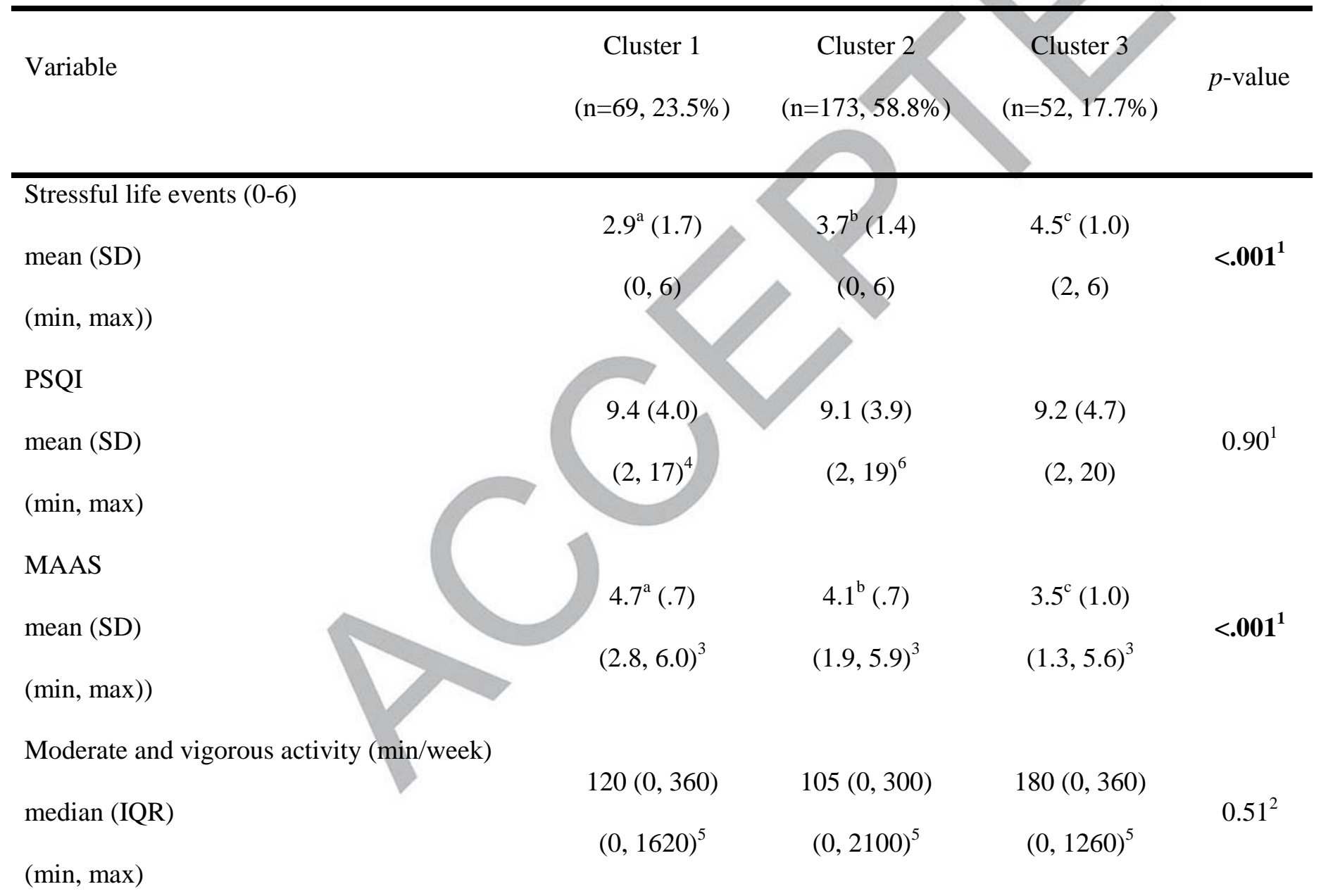


Diagnosed comorbid disorders

median (IQR)

$2(0,3) \quad 2(1,3) \quad 2(1,3)$

$(0,10) \quad(0,11) \quad(0,9)$

$0.91^{2}$

(min, max)

Undiagnosed comorbid symptoms

median (IQR)

$2^{\mathrm{a}}(1,4) \quad 2^{\mathrm{a}}(1,4) \quad 4^{\mathrm{b}}(2,5)$

$(0,9) \quad(0,9) \quad(0,9)$

$\mathbf{0 . 0 0 3}^{2}$

(min, $\max )$

Body chart squares filled in

median (IQR)

$9^{\mathrm{a}}(5,16)$

$13^{\mathrm{a}}(7,20)$

$17^{\mathrm{b}}(11,28)$

(min, $\max )$

$(1,75)$

$(4,62)$

FreBAQ

mean $(\mathrm{SD})$

$5.9^{\mathrm{a}}(4.2) \quad 9.5^{\mathrm{b}}(5.8)$

$(0,18) \quad(0,25)$

(min, max)

\footnotetext{
${ }^{1}$ analysis of variance; ${ }^{2}$ Kruskal-Wallis one-way analysis of variance $;{ }^{3}$ missing in one case; ${ }^{4}$ missing in four cases;

missing in two cases; ${ }^{6}$ missing in seven cases

${ }^{a, b, c}$ Superscripted letters define significantly different groups, i.e. results with different letters are significantly different

PSQI - Pittsburgh Sleep Quality index; MAAS - Mindful Attention Awareness scale
}

Table 4: Tissue sensitivity data for the three clusters derived using latent class analysis

\begin{tabular}{lcccc}
\hline Cluster 1 & Cluster 2 & Cluster 3 & $p$-value \\
Variable & $(\mathrm{n}=69,23.5 \%)$ & $(\mathrm{n}=173,58.8 \%)$ & $(\mathrm{n}=52,17.7 \%)$ & \\
& & & \\
\hline
\end{tabular}

Temporal summation

n $(\%)$
$10(14.7)$
$33(19.1)$
$13(25.0)$

$.36^{1}$ 
PPT wrist $(\mathrm{kPa})$

$$
269.0(191.3,385.3) \quad 268.0(184.0,344.7) \quad 271.0(142.7,330.2)
$$

median (IQR)

$$
(80.0,1060.3) \quad(55.3,1200.0) \quad(57.0,939.0)
$$

(min,max)

PPT lumbar $(\mathrm{kPa})$

$$
283.0^{\mathrm{a}}(197.3,506.7) \quad 281.7^{\mathrm{a}}(168.7,458.7) \quad 178.5^{\mathrm{b}}(95.7,402.0)
$$

median (IQR)

$$
(36.7,1349.0) \quad(37.0,1600.0) \quad(49.0,964.0)
$$

(min,max)

HPT wrist $\left({ }^{\circ} \mathrm{C}\right)$

median (IQR)

$$
45.5(43.7,48.1)
$$

$45.3(42.5,47.8)$

$45.0(41.4,47.6)$

$(34.5,50.0)$

$(33.9,50.0)$

$(32.2,50.0)$

(min,max)

HPT lumbar $\left({ }^{\circ} \mathrm{C}\right)$

mean (SD)

$$
42.4(3.8)
$$

41.5 (4.4)

$(34.6,50.0)$

$(33.6,50.0)$

$(33.6,50.0)$

(min,max)

CPT wrist $\left({ }^{\circ} \mathrm{C}\right)$

median (IQR)

(min,max)

(min,max)

Two-point

discrimination $(\mathrm{cm})$

(min,max)

\footnotetext{
${ }^{1} \chi^{2}$ analysis; ${ }^{2}$ Kruskal-Wallis one-way analysis of variance $;{ }^{3}$ analysis of variance

${ }^{a, b}$ Superscripted letters define significantly different groups, i.e. results with different letters are significantly different PPT - pressure pain threshold; HPT - heat pain threshold; CPT - cold pain threshold; $\mathrm{kPa}$ - kilopascals; ${ }^{\circ} \mathrm{C}$ - degrees centigrade
} 
Table 5: Pain responses to repeated spinal movements, and behaviours associated with pain for the three clusters derived using latent class analysis

\section{$\begin{array}{lll}\text { Cluster } 1 & \text { Cluster } 2 & \text { Cluster } 3\end{array}$}

Variable

$p$-value

$$
(\mathrm{n}=69,23.5 \%) \quad(\mathrm{n}=173,58.8 \%) \quad(\mathrm{n}=52,17.7 \%)
$$

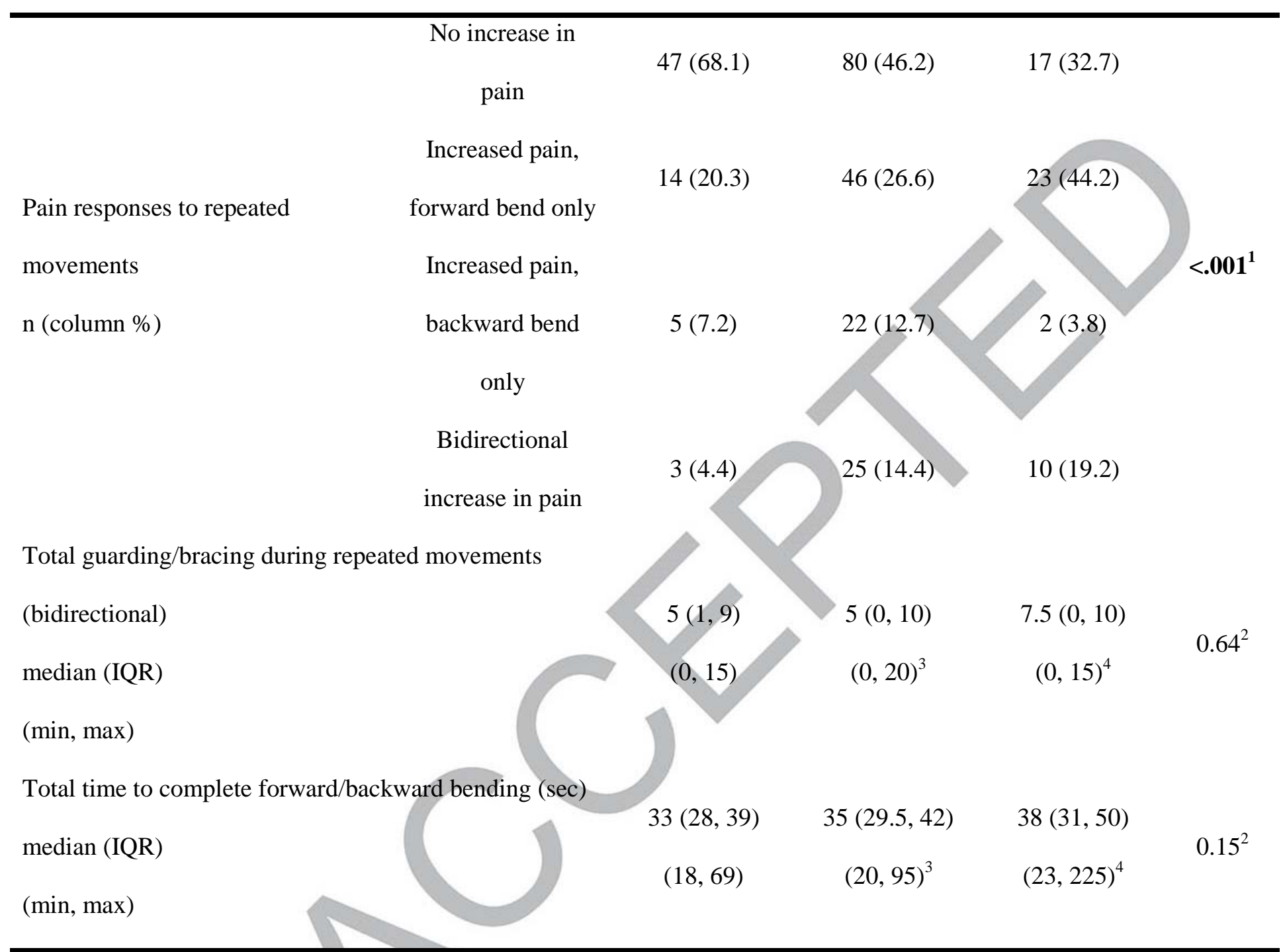

${ }^{1} \chi^{2}$ analysis; ${ }^{2}$ Kruskal-Wallis one-way analysis of variance $;{ }^{3}$ missing in one case $;{ }^{4}$ missing in three cases 